\title{
Influencia de una intervención educativa en el nivel de ansiedad de un paciente en hemodiálisis
}

\author{
Espinoza-Soto Joycee ${ }^{1}$, Jiménez-Moreno Jessica ${ }^{1}$, Salazar-Calderón Katherine ${ }^{1}$, \\ Silva-Mathews Zulema
}

\section{RESUMEN}

Objetivo: determinar la influencia de la intervención de enfermería en la disminución de ansiedad en pacientes en tratamiento con hemodiálisis en el Hospital Nacional Dos de Mayo en el año 2011. Material y métodos: estudio cuasiexperimental, con una muestra conformada por 73 pacientes que cumplieron con los criterios de selección, a los que se le aplico el Test de Zung antes y después del desarrollo de la intervención educativa. Resultados: los niveles de ansiedad de los pacientes antes de la intervención educativa y la terapia de relajación fueron: 36 (49,3\%) presentaron ansiedad leve; 36 (49,3\%) presentaron ansiedad moderada; 1 (1,4\%) presentó ansiedad severa. Después de la intervención de enfermería fueron: 34 (46,6\%) no presentaron ansiedad; $38(52,1 \%)$ presentaron ansiedad leve, y 1 $(1,4 \%)$ presentó ansiedad moderada. Conclusiones: los pacientes hemodializados después de la intervención educativa mostraron una disminución considerable en el nivel de ansiedad moderado. .

Palabras clave: ansiedad, educación en enfermería, hemodiálisis. Perú. (Fuente DeCs BIREME).

\section{Influence of patient education on the anxiety level of a patient on hemodialysis}

\begin{abstract}
Objetive: Determine the influence of nursing intervention in reducing anxiety in patients undergoing hemodialysis treatment at the Hospital Nacional Dos de Mayo in 2011. Material and methods: Quasi-experimental study with a sample consisting of 73 patients who met the selection criteria, to which I apply the Zung test before and after the development of the educational intervention. Results: Anxiety levels of patients before the educational intervention and relaxation therapy were: $36(49.3 \%)$ had mild anxiety, $36(49.3 \%)$ had moderate anxiety, $1(1.4 \%)$ had anxiety severe. After nursing intervention were: $34(46.6 \%)$ had no anxiety, $38(52.1 \%)$ had mild anxiety and $1(1.4 \%)$ had moderate anxiety. Conclusions: Hemodialysis patients after the educational intervention showed a significant decrease in the level of moderate anxiety.
\end{abstract}

Key words: Anxiety, nursing education, hemodialysis. Peru (Source DeCs BIREME).

\section{INTRODUCCIÓN}

Según estudios realizados, los pacientes con insuficiencia renal crónica en tratamiento con hemodiálisis presentan una serie de cambios en el estilo de vida, constantes evaluaciones clínico médicas, posibles recaídas y complicaciones (1).
La hemodiálisis disminuirá los signos y síntomas propios de la enfermedad pero a su vez pueden aparecer complicaciones como respuesta del organismo y así provocar una serie de alteraciones psicológicas como: depresión, angustia, temor, ansiedad, síndrome de adaptación, estrés, entre otras. Los pacientes con insuficiencia renal crónica sometidos a hemodiálisis cursan con frecuencia con an-

\footnotetext{
Egresada de Enfermería de la Universidad Peruana Cayetano Heredia. Lima - Perú.

Magister en Enfermería. Profesora Asociada de la Facultad de Enfermería. Universidad Peruana Cayetano Heredia. Lima - Perú.
} 
siedad, la que influye en la vida familiar y la ocupación de los pacientes, causando dificultades que aumenta los síntomas psicológicos $(2,3)$.

La Organización Mundial de la Salud (OMS) define la salud como "Aquel estado de bienestar físico, psicológico y social, y no solo como la ausencia de enferme$d a d »$. Teniendo en cuenta esta definición, cabe resaltar la importancia del aspecto psicológico de la persona.

En la práctica clínica el estado emocional del paciente muchas veces no es tomado en cuenta, tal vez porque el trabajo en este aspecto no es fácil para el profesional de la salud, debido a las diferentes expresiones, manifestaciones y adaptaciones del paciente, que dependen de las vivencias, historias de enfermedad y características personales.

Es posible que la falta de conocimiento sobre el tratamiento con hemodiálisis y el inadecuado manejo de las emociones contribuyan en la vulnerabilidad del paciente, llegando a establecerse, en algunos casos, niveles de ansiedad que refuerzan la vivencia ya condicionada por el tratamiento y las complicaciones propias de la enfermedad (4).

El profesional de enfermería es responsable del tratamiento hemodialítico, es quien permanece con el paciente y cumple un papel importante durante el proceso de enfermedad y tratamiento, por ello entre las acciones que realiza debe incluir actividades educativas que contribuyan a mejorar la calidad de vida, la capacidad de afrontamiento en el caso de presentar algún nivel de ansiedad, desarrollando las capacidades y habilidades propias del mismo (5).

Por tal motivo, se considera de vital importancia brindar intervenciones de enfermería, de manera oportuna y continua, que contribuyan a disminuir la ansiedad en los pacientes en tratamiento con hemodiálisis.

\section{MATERIAL Y MÉTODOS}

La investigación fue cuasiexperimental realizada en la Unidad de Hemodiálisis del Hospital Nacional Dos de Mayo. La población estuvo conformada por 73 pacientes que cumplieron con los criterios de inclusión y exclusión, y participaron voluntariamente firmando el consentimiento informado.

La técnica utilizada para la recolección de la información fue la encuesta y como instrumento el test de Zung para medir el nivel de ansiedad, conformado por 20 items, de los cuales 15 preguntas son positivas y 5 son negativas (59-13-17-19). Cada una de estas tiene cuatro respuestas alternativas: muy pocas veces, algunas veces, muchas veces, y casi siempre. El puntaje total fue sometido a una fórmula que nos permitió clasificar a los pacientes según su nivel de ansiedad.

Para el desarrollo del estudio se solicitó la autorización al Comité de Ética de la UPCH, dirección y jefatura médica y de enfermería del servicio de hemodiálisis.

Se explicó a los pacientes la finalidad del estudio, su participación voluntaria y anónima y de aceptar participar la firma del consentimiento informado.

El programa se realizó en uno de los ambientes del servicio de hemodiálisis con una duración aproximada de 30 minutos.

\section{RESULTADOS}

El procesamiento y análisis de los datos se realizó usando el paquete estadístico SPSS v. 15, programa de Excel. Para identificar los niveles de ansiedad tanto antes y después de la intervención de enfermería en los pacientes en tratamiento con hemodiálisis, se utilizó tablas de frecuencia univariadas absolutas como porcentuales. Para comparar los niveles de ansiedad antes y después de la intervención de enfermería se aplicó la prueba no paramétrica signo - rango de Wilcoxon.

\section{Gráfico 1. Niveles de ansiedad de los pacientes del servicio de hemodiálisis según el pretest. Hospital Nacional Dos de Mayo - marzo 2011}

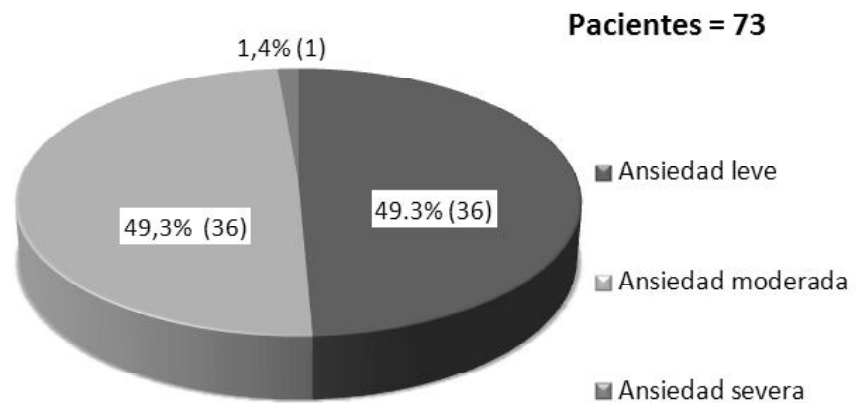

En el grafico 1, se observa que en una población de 73 pacientes $(100 \%)$ en tratamiento con hemodiálisis, los niveles de ansiedad de los pacientes antes de la intervención educativa y la terapia de relajación fueron: 36 (49,3\%) presentaron ansiedad leve; $36(49,3 \%)$ presentaron ansiedad moderada; $1(1,4 \%)$ presentó ansiedad severa. 
Gráfico 2. Niveles de ansiedad de los pacientes del servicio de hemodiálisis según el postest. Hospital Nacional Dos de Mayo - marzo 2011

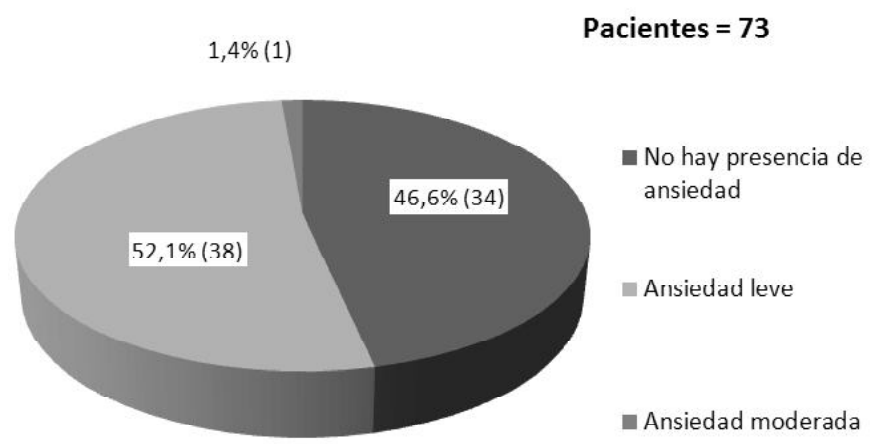

En el grafico 2 se observa que en una población de 73 pacientes $(100 \%)$ en tratamiento con hemodiálisis los niveles de ansiedad de los pacientes después de la intervención de enfermería fueron: $34(46,6 \%)$ no presentaron ansiedad; $38(52,1 \%)$ presentaron ansiedad leve, y 1 $(1,3 \%)$ presentó ansiedad moderada.

En la tabla 1, se observa en el pretest que de los adultos maduros, 19 presentan mayor nivel de ansiedad moderada $(59,3 \%)$, y 1 severa $(3,2 \%)$; a diferencia de los adultos mayores quienes presentan mayor porcentaje de ansiedad leve, 23 pacientes (59\%). En el postest se evidencia que persiste un mayor porcentaje de ansiedad en el adulto

Tabla 1. Niveles de ansiedad según edad de los pacientes del servicio de hemodiálisis del Hospital Nacional Dos de Mayo - Marzo 2011

\begin{tabular}{|c|c|c|c|c|c|c|c|c|c|c|c|c|c|c|c|c|}
\hline \multirow{3}{*}{ Edad } & \multicolumn{6}{|c|}{ Pretest } & \multicolumn{10}{|c|}{ Post est } \\
\hline & \multicolumn{2}{|c|}{ De 18 a 25} & \multicolumn{2}{|c|}{ De 26 a 59} & \multicolumn{2}{|c|}{$\begin{array}{l}\text { De } 60 \text { a } \\
\text { más }\end{array}$} & \multicolumn{2}{|c|}{ Total } & \multicolumn{2}{|c|}{ De 18 a 25} & \multicolumn{2}{|c|}{ De 26 a 59} & \multicolumn{2}{|c|}{ De 60 a más } & \multicolumn{2}{|c|}{ Total } \\
\hline & $\mathbf{N}$ & $\%$ & $\mathbf{N}$ & $\%$ & $\mathbf{N}$ & $\%$ & $\mathbf{N}$ & $\%$ & $\mathbf{N}$ & $\%$ & $\mathbf{N}$ & $\%$ & $\mathbf{N}$ & $\%$ & $\mathbf{N}$ & $\%$ \\
\hline No presenta ansiedad & & & & & & & & & 1 & 50 & 10 & 31,2 & 196 & 48,7 & & \\
\hline Ansiedad leve & 1 & 50 & 12 & 37,5 & 23 & 59 & & & 1 & 50 & 21 & 65,6 & 20 & 51,3 & & \\
\hline Ansiedad moderada & 1 & 50 & 19 & 59,3 & 16 & 41 & 73 & 100 & 0 & 0 & 1 & 3,2 & 0 & 0 & 73 & 100 \\
\hline Ansiedad severa & 0 & 0 & 1 & 3,2 & 0 & 0 & & & 0 & 0 & 0 & 0 & 0 & 0 & & \\
\hline Total & 2 & 100 & 32 & 100,0 & 39 & 100 & & & 2 & 100 & 32 & 100 & 39 & 100,0 & & \\
\hline
\end{tabular}

Tabla 2. Niveles de ansiedad según sexo de los pacientes del servicio de hemodiálisis del hospital del Hospital Nacional Dos de Mayo - Marzo 2011

\begin{tabular}{|c|c|c|c|c|c|c|c|c|c|c|c|c|}
\hline \multirow{3}{*}{ Edad } & \multicolumn{6}{|c|}{ Pretest } & \multicolumn{6}{|c|}{ Postest } \\
\hline & \multicolumn{2}{|c|}{ Hombres } & \multicolumn{2}{|c|}{ Mujeres } & \multicolumn{2}{|c|}{ Total } & \multicolumn{2}{|c|}{ Hombres } & \multicolumn{2}{|c|}{ Mujeres } & \multicolumn{2}{|c|}{ Total } \\
\hline & $\mathbf{N}$ & $\%$ & $\mathbf{N}$ & $\%$ & $\mathbf{N}$ & $\%$ & $\mathbf{N}$ & $\%$ & $\mathbf{N}$ & $\%$ & $\mathbf{N}$ & $\%$ \\
\hline No presenta ansiedad & & & & & & & 21 & 46,7 & 13 & 46,7 & & \\
\hline Ansiedad leve & 21 & 46,7 & 15 & 53,6 & & & 24 & 53,3 & 14 & 50,0 & & \\
\hline Ansiedad moderada & 24 & 53,3 & 12 & 42,9 & 73 & 100 & 0 & 0 & 1 & 3,6 & 73 & 100 \\
\hline Ansiedad severa & 0 & 0 & 1 & 3,6 & & & 0 & 0 & 0 & 0 & & \\
\hline Total & 45 & 100,0 & 28 & 100,0 & & & 45 & 100,0 & 28 & 100,0 & & \\
\hline
\end{tabular}

Tabla 3. Niveles de ansiedad según tiempo de tratamiento con hemodiálisis, de los pacientes del servicio de hemodiálisis del Hospital Nacional Dos de Mayo - Marzo 2011

\begin{tabular}{|c|c|c|c|c|c|c|c|c|c|c|}
\hline \multirow{3}{*}{ E dad } & \multicolumn{4}{|c|}{ Pretest } & \multicolumn{4}{|c|}{ Post est } & \multirow{2}{*}{\multicolumn{2}{|c|}{ Total }} \\
\hline & \multicolumn{2}{|c|}{ Menos de 16 m } & \multicolumn{2}{|c|}{ Mayor de $16 \mathrm{~m}$} & \multicolumn{2}{|c|}{ Menos de $16 \mathrm{~m}$} & \multicolumn{2}{|c|}{ Mayor de $16 \mathrm{~m}$} & & \\
\hline & $\mathbf{N}$ & $\%$ & $\mathbf{N}$ & $\%$ & $\mathbf{N}$ & $\%$ & $\mathbf{N}$ & $\%$ & $\mathbf{N}$ & $\%$ \\
\hline No presenta ansiedad & & & & & 19 & 50 & 15 & 42,9 & 73 & 100 \\
\hline Ansiedad leve & 19 & 50 & 17 & 48,6 & 19 & 50 & 19 & 54,3 & 73 & 100 \\
\hline Ansiedad moderada & 19 & 50 & 17 & 48,6 & 0 & 0 & 1 & 2,9 & 73 & 100 \\
\hline Ansiedad se vera & 0 & 0 & 1 & 2,9 & 0 & 0 & 0 & 0 & 73 & 100 \\
\hline Total & 38 & 100 & 35 & 100,0 & 38 & 100 & 35 & 100 & 73 & 100 \\
\hline
\end{tabular}


Tabla 4. Niveles de ansiedad según pre y postest de los pacientes del servicio de hemodiálisis del Hospital Nacional Dos de Mayo - Marzo 2011

\begin{tabular}{|c|c|c|c|c|c|c|c|c|}
\hline \multirow{2}{*}{ Niveles de ansiedad } & \multicolumn{4}{|c|}{ Pretest } & \multicolumn{4}{|c|}{ Postest } \\
\hline & $\mathbf{N}$ & $\%$ & $\mathbf{N}$ & $\%$ & $\mathbf{N}$ & $\%$ & $\mathbf{N}$ & $\%$ \\
\hline No hay presencia de ansiedad & 0 & 0 & \multirow{4}{*}{73} & \multirow{4}{*}{100} & 34 & 46,6 & \multirow{4}{*}{73} & \multirow{4}{*}{100} \\
\hline Ansiedad leve & 36 & 49,3 & & & 38 & 52,1 & & \\
\hline Ansiedad moderada & 36 & 49,3 & & & 1 & 1,3 & & \\
\hline Ansiedad severa & 1 & 1,4 & & & 0 & 0 & & \\
\hline
\end{tabular}

maduro, encontrando ansiedad leve en 21 pacientes $(65,6 \%)$, moderada en $1(3,2 \%)$, en comparación al adulto mayor que presenta ansiedad leve en 20 pacientes $(51,3 \%)$ y no presenta ansiedad en $19(48,7 \%)$.

En la tabla 2 se observa en el pretest que 24 hombres presentan ansiedad moderada (53,3\%); 21 ansiedad leve $(46,7 \%)$; a diferencia de las mujeres, 15 presentan ansiedad leve (53,6\%); 12 ansiedad moderada (42,9\%); y 1 ansiedad severa $(3,6 \%)$, En el postest se encuentra cambios: 24 hombres presentan ansiedad leve $(53,3 \%)$ y 21 no presentan ansiedad (46,7\%); 14 mujeres presentan ansiedad leve (50\%), 13 no presentan ansiedad $(42,4 \%)$ y 1 moderada $(3,6 \%)$, no evidenciándose la ansiedad severa.

En la tabla 3, en el pretest realizado a los pacientes que asisten al tratamiento con hemodiálisis en un tiempo menor de 16 meses, se observa que tanto la ansiedad leve como la moderada presenta el mismo resultado, leve en 19 pacientes (50\%); moderada en 19 pacientes (50\%); entre los pacientes con un tiempo mayor de 16 meses, 17 presentan ansiedad leve (48,6\%), 17 moderada $(48,6 \%)$ y, además, 1 ansiedad severa $(2,9 \%)$, En el postest los pacientes en tratamiento menor de 16 meses, 19 presentan ansiedad leve (50\%); 19 no presentan ansiedad (50\%); de los pacientes en tratamiento mayor de 16 meses 19 presentan ansiedad leve $(54,3 \%)$; 15 no presentan ansiedad $(42,9 \%)$ y 1 ansiedad moderada $(2,9 \%)$,

En la tabla 4, según la Prueba de Wilcoxon, se concluye que la intervención de enfermería influye en la disminución de los niveles de ansiedad de los pacientes que acuden a la unidad de hemodiálisis del HNDM (prueba de Wilcoxon, $\mathrm{p}<0,001)$.

\section{DISCUSIÓN}

La enfermedad renal crónica y el tratamiento con hemodiálisis son sucesos vitales, que llevan al paciente a presentar algún nivel de ansiedad, él deberá adaptarse a la nueva situación para lograr algún nivel de equilibrio emocional. Por ello, consideramos importante incorporar en el tratamiento contenidos educativos y sesiones de relajación de manera continua.

El objetivo del estudio fue determinar la influencia de la intervención de enfermería en la ansiedad presente en los pacientes en tratamiento con hemodiálisis en el Hospital Nacional Dos de Mayo.

Los resultados encontrados en el presente estudio, en la etapa del pretest, evidencian que el $100 \%$ de los pacientes presenta algún nivel de ansiedad; leve el 49,3\%; moderada el 49,3\%, y severa el 1,4\% (gráfico 1).

En estudios realizados, como el de Escobar M., Perea O., titulado «Ansiedad y depresión en relación al tiempo de tratamiento en hemodiálisis» (6) se presentó resultados similares, donde el $88,9 \%$ de la muestra presentó algún nivel de ansiedad, no encontrándose nivel de ansiedad severa.

La diferencia que se establece entre ambas poblaciones de estudio probablemente se deba a que una de ellas es asegurada y la otra no. Contar con un seguro permite al paciente mantener su tratamiento sin necesidad de recurrir a un gasto extra; en tanto, quienes no lo poseen se encuentran no solo afectados por la enfermedad sino, muchas veces, por la incertidumbre de no contar con los medios económicos para afrontar su tratamiento y los adicionales que se dan; ya sea por la adquisición de un nuevo equipo para su hemodiálisis porque venció en su tiempo.

La población de estudio es de medianos a bajos recursos que no cuentan con los medios económicos que exigen este tratamiento, el cual demanda no solo el costo, sino el apoyo terapéutico adicional, como son las inyecciones de fierro, eritropoyetina postratamiento en razón a su anemia crónica, entre otros. 
Novel G. considera que las causas de la ansiedad se pueden buscar tanto en componentes internos o propios de la persona como en su entorno social y ambiental. Dentro de los factores sociales considera como elementos estresantes los económicos, el desempleo, pagos imprevistos, cambios bruscos de ingresos y egresos, etc. (7).

Así también, se evidencia una similitud de resultados entre el nivel de ansiedad leve $(49,3 \%)$ y el de ansiedad moderada $(49,3 \%)$; la que estaría asociada a las respuestas de la persona en sus diferentes dimensiones fisiológicas, cognitivas y conductuales.

Es así que las personas pueden responder ante la enfermedad de diferentes formas, las que pueden estar relacionadas con aspectos sociales, laborales y familiares, que a su vez responden a la necesidad de apoyo emocional y a las condiciones necesarias para mantener no solo el tratamiento sino, en la mayoría de los casos, tratamiento de enfermedades primarias como la diabetes, hipertensión arterial, cardiopatías, lupus eritematoso, entre otros.

Se puede considerar la edad como otro factor que influye en la ansiedad, ya que en el estudio se observó que los pacientes menores de 60 años presentan mayor porcentaje de ansiedad moderada y severa, a diferencia que los adultos mayores donde prevalece la ansiedad leve (tabla $1)$.

Es posible que los pacientes mayores cuenten con un nivel de afrontamiento que les posibilite aceptar la nueva realidad, porque en su mayoría han realizado muchas de sus expectativas; a diferencia de los más jóvenes, ya que ingresar al tratamiento con hemodiálisis interfiere en sus aspiraciones laborales, económicas, familiares y sociales (8).

Por otro lado Amelia E. Páez en su estudio «Ansiedad y depresión en pacientes con insuficiencia renal crónica en tratamiento con diálisis» expone que con respecto a la edad, mientras mayor edad tienen los enfermos renales crónicos, los niveles de ansiedad estado y depresión aumentan, señalando que en estos pacientes, al progresivo deterioro físico de la vejez se suman las limitaciones inherentes a la enfermedad renal crónica y su tratamiento médico, llevando así a una disminución en las actividades cotidianas, sociales y laborales, lo que se relaciona con la visión negativa de sí mismo (9).

El sexo podría relacionarse con los resultados encontrados; las mujeres presentaron un mayor porcentaje de ansiedad leve a diferencia de los varones que presentaron un mayor porcentaje de ansiedad moderada (tabla 2).

La ansiedad en los varones podría estar relacionada con el rol que desempeñan en la familia, creando un sentimiento de presión en algunos casos, por su responsabilidad en el mantenimiento familiar y el afrontamiento económico además del gasto de su enfermedad, lo que propiciaría un nivel de ansiedad moderado en relación a las mujeres que se encuentran en un nivel de ansiedad leve.

Con respecto al tiempo de tratamiento con hemodiálisis se observo que tanto los pacientes con menos de 16 meses como aquellos con más de 16 meses de tratamiento presentaron algún nivel de ansiedad. (tabla 3) Por lo que podemos decir que ambos grupos requieren de apoyo para afrontar la enfermedad y tratamiento.

El estudio desarrollado por Begoña Ruiz, A., et al., titulado «Cambios en las estrategias de afrontamiento en los pacientes de diálisis a lo largo del tiempo», llegó a la conclusión que conforme trascurre el tiempo en tratamiento con diálisis las personas utilizan menos la distracción y el apoyo social; buscan con mayor intensidad estrategias como el aislamiento social y la confrontación, todo esto disminuye el bienestar psicológico (10).

A su vez las complicaciones del tratamiento propias de la enfermedad se van agravando con el tiempo, y los pacientes perciben una forma de vida más limitada, lo cual puede afectar su estado emocional y, de esta manera, repercutir en su nivel de ansiedad.

Fornés Vives define la ansiedad como un hecho habitual una experiencia universal en la vida. Representa una respuesta o grupo de respuestas a cualquier cambio al que la persona tenga de adaptarse (11).

La ansiedad leve se caracteriza principalmente por el estado de alerta en el que la percepción y la atención de la persona están incrementadas; sin embargo, la persona conserva la capacidad para afrontar y resolver situaciones problemáticas, a diferencia de la ansiedad moderada que provoca disminución de la atención y percepción, dificulta la concentración y disminuye la capacidad para analizar la realidad de forma objetiva (7).

Los niveles de ansiedad podrían estar vinculados a que su vida depende de una máquina, ingiere medicación de por vida y está permanentemente asistiendo a controles médicos en las diferentes especialidades, ya que su enfermedad se va complicando y el deterioro físico y mental se va evidenciando cada vez más, lo cual nos da una señal 
de que el paciente necesita constante apoyo de parte su familia y del personal de enfermería que lo atiende para disminuir el riesgo de trastornos emocionales más significativos.

Por ello, es posible que el apoyo de la familia sea un factor que influye en la presencia de ansiedad ya que constituye un apoyo importante durante el proceso de la enfermedad y tratamiento; sin embargo, se pudo observar que algunos pacientes acudían a sus sesiones de diálisis solos y algunos acompañados por un familiar. Asimismo, se evidenció que los pacientes sentían que eran una carga para su familia, vivían momentos estresantes y, al encontrarse solos, tenían que realizar sus trámites y consultas, y a la vez recibir su tratamiento.

Diversos estudios han mostrado que las personas que disponen de un sistema de apoyo familiar efectivo soportan mejor las crisis de la vida; asimismo, el impacto del estrés es mucho menor que en las personas que no tienen este tipo de apoyo disponible (7).

Indudablemente, la familia es un pilar importante en el cuidado integral del enfermo terminal; hasta tal punto que sin su participación activa es difícil alcanzar los objetivos terapéuticos, pues con su actitud y comportamiento pueden facilitar o bloquear los esfuerzos del paciente por adaptarse y soportar la enfermedad.

Por ello se debe movilizar todos los recursos familiares para utilizarlos como arma terapéutica y, a la vez, apoyar a la familia a soportar el impacto provocado por la enfermedad terminal del ser querido y la carga física, emocional y económica que provoca su atención y cuidado (paciente terminal).

Agregado a esto se evidenció la falta de información. Esto se observó en la población estudiada que no cuenta con los cuidados propios del tratamiento, muchos de ellos por falta de información y otros por incumplimiento. Algunos de los pacientes cuando llegaban al servicio presentaban diferentes complicaciones como edema, hipertensión, calambres, cefaleas, etc, lo cual alteraba su estado emocional mostrándose preocupados y ansiosos; a la vez mostraban inadecuada higiene personal y de fístula arteriovenosa y catéter venoso central.

Según Avendaño, el cumplimiento es inestable y cíclico. Las transgresiones más comunes son las de la dieta, especialmente la ingesta de líquidos, y de la medicación, el calcio entre otros (8). La falta de sesiones de diálisis es menos común e indica problemas más serios (depresión, negación extrema, etc).

El grado de incumplimiento del tratamiento también depende de la comprensión y aceptación que tenga el paciente, y de la convicción de que su participación influye en el resultado. Las carencias en este sentido pueden suplirse con intervenciones (educativas y psicoterapéuticas) del equipo y con apoyo familiar. Son efectivos los programas de educación a pacientes y familiares, y la reflexión con ellos sobre las dificultades de cumplimiento observadas (8).

Gail considera que la educación sanitaria es relevante para la atención de los pacientes con trastornos psicopatológicos. Estos pacientes suelen necesitar información sobre los medicamentos, tratamientos y los cambios de estilo de vida. El paciente y la familia necesitarán información sobre el fomento de la salud mental, los cuidados de seguimiento y el manejo de la crisis, y educación sobre las maneras de afrontar la ansiedad (12).

En relación al postest podemos decir que los niveles de ansiedad disminuyeron significativamente encontrando los siguientes resultados; no hay ansiedad 46,6\%; ansiedad leve $52,1 \%$, y ansiedad moderada $1,4 \%$, (tabla 2). Dichos resultados se lograron con las charlas consecutivas y terapias de relajación. De la misma forma, el estudio realizado por Ampuero P., «Programa congnitivo - conductual para pacientes con IRCT que inician tratamiento con hemodiálisis» encontró que el $100 \%$ de su muestra presentó ansiedad severa durante el pretest, y después de aplicar la terapia de relajación su estado de ansiedad disminuyo: ansiedad leve $78 \%$ y ansiedad moderada $22 \%$.

Durante la intervención educativa se observó que los pacientes no contaban con los conocimientos necesarios sobre su enfermedad, tratamiento y cuidados, pero mostraron interés y participaron activamente durante el programa educativo y en las sesiones de terapias de relajación; lo que refuerza la idea que es importante brindar una educación integral, clara, concisa y de manera periódica, favoreciendo la motivación y participación en su autocuidado y, a la vez, contribuir en el manejo de su ansiedad.

La educación para la salud no solo busca informar al paciente sobre la enfermedad y los tratamientos sino que va más allá de esas medidas; la educación debería conducir al paciente a modificar sus comportamientos durante el periodo de aprendizaje, y en consecuencia, ayudarlo a adoptar nuevos hábitos de vida, teniendo en cuenta que el paciente posee la capacidad de reflexionar y de actuar, lo 
cual le permitirá controlar su pensamiento, sus sentimientos, sus motivaciones y sus acciones (13).

Durante la revisión bibliográfica se ha podido encontrar que uno de los aportes directos de las teorías cognitivo conductuales respecto a la relajación, postuladas por Meinchembaun, es el de la facilitación del cambio de conducta mediante la restructuración de los pensamientos conscientes, se ha descubierto que ese tipo de terapia ha sido efectiva para el manejo y control de la ansiedad. Es justamente en este punto que se observa la directa relación entre la técnica utilizada (terapia de relajación).

Además, las diversas investigaciones evidencian que proporcionar información, siempre que se haga en un forma precisa, facilita la adaptación y colaboración del enfermo en su proceso terapéutico, aumenta sus niveles de satisfacción con los cuidados recibidos y, al mismo tiempo, evita la «conspiración de silencio», pero debe hacerse de forma pautada, progresiva, individualizada, siguiendo paso a paso las distintas etapas por las que están pasando el enfermo y su familia, proporcionando la información necesaria y adecuada para cada uno de los momentos con los que ha de enfrentarse, buscando la congruencia dentro del equipo hospitalario y dentro de la familia y buscando siempre el lado positivo de la misma, es decir, manteniendo en todo momento una puerta abierta a la esperanza, aunque al informar al paciente de la gravedad de su enfermedad, se debe hablar con realismo, sinceridad, respeto, entereza y sin dramatizar (12).

Como se puede observar en el pretest la totalidad de pacientes presentó algún nivel de ansiedad, la cual disminuyó una vez concluidas las actividades programadas, evidenciándose esto en el postest.

Pero si bien entendemos que la ansiedad es necesaria para enfrentarnos de forma adecuada a los acontecimientos diarios, esta se considera patológica cuando interfiere en esta capacidad adaptativa e impide llevar adelante las tareas cotidianas, cuando produce sentimientos de inseguridad y de devaluación personal, y dificulta el proceso de relación y de adaptación al medio (14).

Cuando un paciente padece una enfermedad, especialmente si es crónica, la educación y formación sobre aspectos claves de su enfermedad contribuye a mejorar los resultados clínicos en el manejo de su problema de salud (15), es así que la enfermera, en su rol de educadora, debe brindar un cuidado individualizado considerando el aspecto emocional del paciente, utilizando las herramientas necesarias para conservar su estado de salud y mejo- rar su calidad de vida.

No se trata de que no se vuelva a sentir ansiedad en la vida (cosa imposible incluso en personas «sanas») sino de manejarla. Con la práctica y el tiempo, la ansiedad dejará de ser un problema y su aparición será cada vez menos intensa y espaciada en el tiempo.

Se mostró la disminución de la ansiedad a través de la comparación de los resultados del pretest al postest; la ansiedad severa bajó de 1,4 a 0\%, la ansiedad moderada de 49,3 a 1,4\%, la ansiedad leve de 49,3 a $52,1 \%$, y que, finalmente el $46,6 \%$ no presentan ansiedad en el postest (tabla 4), lo cual permite establecer que una intervención educativa y la terapia de relajación influyen en disminuir el nivel de ansiedad en los paciente con tratamiento de hemodiálisis.

\section{REFERENCIAS BIBLIOGRÁFICAS}

1. García Valderrama F. Mala Adherencia con la terapia Hemodialítica: Rol de la sintomatología Ansiosa y Depresiva. Hospital Nacional Arzobispo Loayza, Centro de hemodiálisis Santa Ana y SERMEDES. [tesis].Lima: Universidad Peruana Cayetano Heredia. FMAH. 2001.

2. Atencio BJ, Nucette E, Colina J, Sumalave S, Gómez F. \& Hinestroza D. (2004). Evaluación de la depresión y ansiedad en pacientes con insuficiencia renal crónica sometidos a hemodiálisis. Archivos Venezolanos de Psiquiatría y Neurología, 50(103), 35-41. 2004.

3. Piazza Alfredo R. Sociedad Peruana de Nefrología. [citado 18 Nov 2011] Disponible en: http://www.spn. org.pe/modules/news/article.php?storyid=2

4. Registro Nacional de Hemodiálisis (RENDE) [citado 17 Nov 2011] Disponible en: http://www.essalud. gob.pe/empresarial/salud/boltecnol20.pdf

5. Andrés Galache Belén. Diagnósticos de enfermería en pacientes con insuficiencia renal crónica en hemodiálisis. Rev Soc Esp Enferm Nefrol [revista en la Internet]. 2004 Sep [citado 2011 Fev 13] ; 7(3): 158-163. Disponible en: http://scielo.isciii.es/ scielo.php?script=sci_arttext\&pid=S1139-1375200 4000300003\&lng=es. http://dx.doi.org/10.4321/ S1139-13752004000300003.

6. Escobar Mamani, Elva; Perea Olivar, Gladys. Ansiedad y depresión en relación al tiempo de tratamiento de hemodiálisis. Centro de hemodiálisis del cono norte.[tesis para licenciatura].Lima: Universidad Peruana Cayetano Heredia. 2005. 
7. Novel M.; Luch G.; Lopez de Vergara D. Enfermería Psicosocial y Salud Mental. España, Masson. Pág. 83-84, 166-167, 240-241. 2005.

8. Avendaño Hernando L. Nefrología Clínica, 2da. Edición. Madrid- España, Editorial médica Panamericana, S.A. Pág. 806-808. 2004.

9. Paez Amelia, Jofré Marcos J., Azpiroz Carmen R., De Bortoli Miguel Ángel. Ansiedad y depresión en pacientes con insuficiencia renal crónica en tratamiento con diálisis. [citado 22 Ago 2011] Disponible en : http://sparta.javeriana.edu.co/psico logia/publicaciones/actualizarrevista/archivos/ V08N01A09.pdf.

10. Begoña Ruiz de Alegría Fernández de Retana, Nekane Basabe Barañano, Encarnación Fernández Prado, Consuelo Baños Baños, María Ángeles Nogales Rodríguez, Miren Echebarri Escribano, J. San Vicente Sancho, Ana Bejarano García. Cambios en las estrategias de afrontamiento en los pacientes de diálisis a lo largo del tiempo. [citado 17 Nov 2011]. Disponible en: http://www.revistaseden.org/files/ 2083_cambios.pdf

11. Fornes Vives J. Enfermería de salud mental y psiquiatría. España. Medico Panamericana. Pág. 37 $-39.2006$.

12. Gail W. Stuart, Michele T. Laraia. Enfermería psiquiátrica. 8va Edición. España - Madrid, Editorial Elsevier. Pág. 297-298. 2006.
13. García Garrido Ll; Baltasar Bague A. La educación para la salud: Adopción de actitudes y mantenimiento de comportamientos saludables. [citado 10 Mar 2010]. Disponible en: http://www.enfermeriaencardiologia. com/publicaciones/manuales/preven/cap_02_ sec_02.pdf

14. Rigol CA, Ugolde AM. Enfermería en salud mental y psiquiatría. España, Editorial MCMXCI MASSON, S.A. Pag.126. 2006.

15. Sáez Rodríguez Loreto, Pérez Paz M. Jesús Valiño Pazos Cristina, Castro Fernández Silvia. Análisis y evaluación de la enfermera referente del paciente en hemodiálisis. Rev Soc Esp Enferm Nefrol [revista en la Internet]. 2009 Jun [citado 2012 Jul 26] ; 12(2): 16-20. Disponible en: http://scielo.isciii.es/ scielo.php?script $=$ sci_arttext $\&$ pid $=$ S 1139 13752009000200003\&lng=es. http://dx.doi.org/ 10.4321/S1139-13752009000200003.

\section{Correspondencia}

Zulema Silva Mathews

Miguel Baquero 251, Lima.

Telefóno: 4314731 - 4244731

Correo electrónico: zulema.silva@upch.pe

Forma de citar este artículo: Espinoza-Soto J, Jiménez-Moreno J, Salazar-Calderón K, Silva- Mathews Z. Influencia de una intervención educativa en el nivel de ansiedad de un paciente en hemodiálisis. Rev enferm Herediana. 2013;6(1):34-41. 